\title{
Perfiles motivacionales, implicación y ansiedad ante los deberes escolares y rendimiento académico en estudiantes de Educación Secundaria Obligatoria
}

\author{
Bibiana Regueiro", Susana Rodríguez*, Isabel Piñeiro*, Carlos Freire*, Mar Ferradás", Elena Gayo** y Antonio Valle*
}

*Universidade da Coruña. ${ }^{* *}$ Universidade de Vigo.

\begin{abstract}
Resumen
El propósito de este trabajo es identificar estudiantes con distintos perfiles motivacionales y analizar las diferencias en su implicación en los deberes escolares y en la ansiedad ante ellos y en el rendimiento académico. La muestra es de 714 estudiantes de Secundaria. De los grupos identificados el de metas de aprendizaje realiza más cantidad de deberes, dedica más tiempo a ellos y gestiona mejor ese tiempo y obtiene un mejor rendimiento. El grupo de evitación del fracaso tiene niveles altos de ansiedad ante los deberes. El grupo de estudiantes desmotivados presenta baja implicación en los deberes, bajo rendimiento y niveles bajos de ansiedad antes los deberes.
\end{abstract}

Palabras clave: perfiles motivacionales, múltiples metas, deberes escolares, rendimiento académico, Educación Secundaria.

\section{Abstract}

The purpose of this paper is to identify students with different motivational profiles and analyze the differences in their involvement and anxiety about homework and academic achievement. The sample is 714 high school students. Focusing on the groups identified, the group of learning goals takes more amount homework, spends more time, manages better the time and gets better achievement. The group avoidance of failure has high levels of homework anxiety. The group of unmotivated students has low homework engagement, poor achievement and low levels of homework anxiety.

Keywords: motivational profiles, multiple goals, homework, academic achievement, secondary education.

\section{Introducción}

En los últimos años, una parte importante de la investigación motivacional en contextos educativos ha dirigido sus esfuerzos a estudiar cómo los individuos pueden perseguir metas múltiples al mismo tiempo. A pesar de que las teorías acerca de las metas académicas consideraron generalmente que las metas de aprendizaje y las metas de rendimiento eran mutuamente excluyentes, la perspectiva de las múltiples metas asume que los estudiantes pueden perseguir más de una meta dentro de su proceso de aprendizaje (Suárez, Cabanach, y Valle, 2001; Valle et al., 2003; Valle et al., 2013).

Así, por ejemplo los estudiantes pueden perseguir metas de aprendizaje, metas sociales y metas rendimiento de modo combinado, y estas pueden estar en armonía o en conflicto e incluso son susceptibles de ir cambiando a lo largo del tiempo y en función del contexto. Sin embargo, todavía existen muchas dudas sobre cómo se articulan e integran diferentes metas dentro de un mismo estudiante. Una de ellas tiene que ver con saber si los estudiantes combinan las metas de manera simultánea o si, por el contrario, hay una alternancia entre ellas ante una tarea concreta (Harackiewicz y Linnerbrlik, 2005)

Es evidente que la perspectiva de las múltiples metas ha contribuido a que los investigadores contemplaran una diversidad más amplia de motivos que tienen los estudiantes para implicarse en la escuela (por ejemplo, obtención de recompensas, evitación de castigos, necesidad de lograr el respecto de otros, necesidad de valoración social, evitación del rechazo de los otros significativos, etc.), suponiendo que las metas académicas y las metas sociales no son independientes, sino que tienen efectos recíprocos. Así, por ejemplo, tener metas sociales y relaciones exitosas entre compañeros puede fomentar que los alumnos se impliquen más en el proceso de enseñanza y aprendizaje y obtengan mejores resultados (Mestre, Samper, Tur, Cortés y Nácher, 2006; Wentzel, 2001). Quizás el mayor problema para el estudiante sea seleccionar y priorizar esas metas y luego coordinarlas de modo efectivo (Dweck, 2001).

Una gran parte de las relaciones entre las variables motivacionales implicadas en el aprendizaje se han estudiado tomando como referente las tareas y actividades que lleva a cabo el aprendiz dentro del aula. Sin embargo, existe un cierto desconocimiento de las relaciones que se producen entre estas variables a lo largo del proceso de realización de los deberes escolares. Según Zimmerman, Bonner y Kovach (1996), para tener éxito en la realización de los deberes escolares, los estudiantes deben ser autorregulados estableciendo los objetivos de los deberes, eligiendo las estrategias de aprendizaje adecuadas, manteniendo la motivación, supervisando los progresos y evaluando los resultados. Así, de modo similar a lo que sucede en el contexto del aula, los estudiantes se enfrentan a los deberes escolares teniendo como referentes las razones y motivos por los que hacen los deberes así como las metas que persiguen al realizarlos.

Bajo estos planteamientos, este trabajo tiene dos propósitos fundamentales. El primero, comprobar si existen combinaciones de metas académicas y no académicas que den lugar a diferentes perfiles 
motivacionales (definidos en función del peso que tienen cada una de las metas dentro de cada perfil) en los estudiantes de secundaria. Una vez hallados y definidos los perfiles motivacionales, el segundo propósito consiste en indagar si existen diferencias entre los grupos obtenidos en algunas variables relevantes referidas a la implicación de los estudiantes en los deberes escolares, a la ansiedad que les provocan esos deberes y a su rendimiento académico.

\section{Participantes}

\section{Método}

En el estudio participaron 714 estudiantes (43.4\% chicos y $56.6 \%$ chicas) de Educación Secundaria Obligatoria (ESO) pertenecientes a 14 centros de tres provincias del norte de España. Del total de participantes el $26.6 \%$ cursaba $1^{\circ}$ ESO; el $20.8 \%$ cursaba $2^{\circ} \mathrm{ESO}$; el $24.9 \%$ cursaba $3^{\circ}$ ESO y el $27.7 \%$ cursaba $4^{\circ}$ ESO.

\section{Instrumentos}

- Las metas académicas se han evaluado mediante el Cuestionario para la Evaluación de Metas Académicas en Secundaria (CEMA-II). Este instrumento, elaborado por Núñez, González-Pienda, González-Pumariega, García y Roces (1997), permite diferenciar los siguientes tipos de metas: metas de implicación en el estudio para la adquisición de competencia y control, metas de implicación en el estudio por el interés en las materias, metas de implicación en el estudio para una defensa del yo -metas de evitación del rendimiento-, metas de evitación del trabajo para una defensa del yo, metas de implicación en el estudio para una búsqueda de engrandecimiento del yo -metas de aproximación al rendimiento-, metas de implicación en el estudio para la adquisición de valoración social, metas de implicación en el estudio por el deseo de obtener un trabajo futuro digno y metas de implicación en el estudio para evitar castigos.

- Para medir las variables vinculadas con la implicación en los deberes escolares y la ansiedad ante los deberes se utilizó la Encuesta sobre los Deberes Escolares (EDE), que es una escala que evalúa diferentes dimensiones relativas a la eficacia de los deberes para el aprendizaje y el rendimiento académico de los alumnos (véase, p. e. Núñez et al., 2013; Núñez et al., 2015; Pan et al., 2013; Rosário et al., 2009; Valle et al., 2015).

- La evaluación del rendimiento académico medio se obtuvo mediante las calificaciones académicas finales obtenidas por los alumnos participantes en Lengua Española, Lengua Inglesa, Matemáticas y Ciencias Sociales. El rendimiento medio se calculó a través del promedio de las calificaciones en las materias mencionadas.

\section{Procedimiento}

Los datos referidos a las variables objeto de estudio fueron recogidos durante el horario escolar previo consentimiento del equipo directivo y de los profesores de los alumnos. Los cuestionarios se aplicaron en un único momento temporal por personal especializado que colaboró en la investigación.

\section{Análisis de datos}

Los perfiles motivacionales se han definido a partir de las diferentes combinaciones de los ochos tipos de metas que mide el CEMA-II. Siguiendo las recomendaciones de Hair, Anderson, Tatham y Black (1999), se utilizó un análisis de conglomerados (mediante el procedimiento no jerárquico K-medias).

Una vez definidos los perfiles motivacionales, se realizó un análisis multivariado de varianza (MANOVA), contemplando como variables dependientes la cantidad de deberes realizados, el tiempo dedicado a los deberes, el aprovechamiento del tiempo dedicado a los deberes, la ansiedad ante los deberes y el rendimiento académico. Como variable independiente se tomaron los conglomerados de procedencia (cuatro niveles o grupos).

Como medida del tamaño del efecto se ha utilizado el coeficiente eta-cuadrado parcial $\left(\eta_{\mathrm{p}}^{2}\right)$, ya que es uno de los procedimientos más utilizados habitualmente dentro de la investigación educativa (ver, p.e., Sun, Pan y Wang, 2010). Para la interpretación de los tamaños del efecto se utiliza el criterio establecido en el trabajo clásico de Cohen (1988), en base al cual, un efecto es pequeño cuando $\eta_{\mathrm{p}}{ }^{2}=.01(d=.20)$, el efecto es medio cuando $\eta_{\mathrm{p}}^{2}=.059(d=.50)$ y el tamaño del efecto es grande si $\eta_{\mathrm{p}}^{2}=.138(d=.80)$.

\section{Perfiles motivacionales}

\section{Resultados}

Los resultados del análisis de conglomerados permitieron identificar cuatro grupos de perfiles motivacionales con diferentes combinaciones de metas. Un primer grupo, integrado por 213 estudiantes (29.8\% de los participantes), presenta puntuaciones altas en todas las metas evaluadas y se define como grupo de estudiantes con múltiples metas.

Un segundo grupo, integrado por 129 estudiantes (18.1\% de los participantes), se caracteriza por bajas puntuaciones en cada una de las metas evaluadas. Se define como el grupo de estudiantes desmotivados.

El tercer grupo, integrado por 167 estudiantes $(23.4 \%$ de los participantes), se caracteriza por un perfil motivacional con predominio de metas de aprendizaje (tanto aquellas orientadas hacia la adquisición de competencia y control como las enfocadas hacia el interés en las materias) y de metas para la obtención de un trabajo futuro digno. Este grupo se ha definido por un perfil motivacional orientado hacia metas de aprendizaje.

Por último, el cuarto grupo, integrado por 205 estudiantes (28.7\% de los participantes), muestra un predominio de metas de implicación en el estudio para una defensa del yo (metas de evitación del rendimiento), de metas de evitación del trabajo para una defensa del yo y de metas de implicación en el estudio para evitar castigos. Este grupo se define por un perfil motivacional orientado a la evitación del fracaso.

Diferencias entre los perfiles motivacionales en la implicación en los deberes, en la ansiedad ante los deberes y en el rendimiento académico

Los resultados reflejaban la existencia de diferencias estadísticamente significativas intergrupo en el conjunto de variables dependientes analizadas $\left(\lambda_{\text {Wilks }}=.706, F\right.$ 
$\left.(15,1938.31)=17.38 ; p<.001, \eta_{\mathrm{p}}^{2}=.110\right)$. El tamaño del efecto es medio. Además, estas diferencias entre los perfiles motivacionales también son significativas para cada una de las variables dependientes contempladas de manera individual. Así, hay diferencias estadísticamente significativas entre los perfiles motivacionales en la cantidad de deberes realizados $(F(3,710)=49.17, p<$ $\left..001, \eta_{\mathrm{p}}{ }^{2}=.173\right)$, en el tiempo dedicado a los deberes $(F$ $\left.(3,710)=13.33, p<.001, \eta_{\mathrm{p}}^{2}=.054\right)$, en el aprovechamiento del tiempo dedicado a los deberes $(F$ $\left.(3,710)=29.30, p<.001, \eta_{\mathrm{p}}^{2}=.111\right)$, en la ansiedad ante los deberes $\left(F(3,710)=21.45, p<.001, \eta_{\mathrm{p}}{ }^{2}=.084\right)$ $\mathrm{y}$ en el rendimiento académico $(F(3,710)=26.38, p<$ $\left..001, \eta_{\mathrm{p}}{ }^{2}=.101\right)$. El tamaño del efecto es grande para la cantidad de deberes, pequeño para el tiempo dedicado a los deberes y medio para las otras variables.

\section{Discusión}

Los resultados de este trabajo permiten confirmar la existencia de perfiles motivacionales a partir de diferentes combinaciones de metas. Concretamente, se han identificado cuatro grupos de perfiles motivacionales distintos: un primer grupo formado por estudiantes con múltiples metas (Grupo de Múltiples Metas), un segundo grupo integrado por estudiantes desmotivados (Grupo de Estudiantes Desmotivados), un tercer grupo formado por estudiantes con predominio de metas de aprendizaje (Grupo de Metas de Aprendizaje) $\mathrm{y}$, por último, un cuarto grupo en el que estaría aquellos estudiantes con un alto miedo al fracaso (Grupo de Evitación del Fracaso).

Con respecto a las diferencias entre los grupos, los resultados indicaron que el perfil de estudiantes orientados hacia metas de aprendizaje es el que presenta unas puntuaciones más altas en todas las variables estudiadas excepto en la ansiedad ante los deberes. Este grupo realiza más cantidad de deberes, pasa más tiempo dedicado a ellos, gestiona mejor ese tiempo y, además, obtiene un rendimiento académico más alto. Unos resultados muy parecidos los obtiene también el grupo de estudiantes con múltiples metas, pero quizás la implicación en los deberes escolares sea más "tranquila" en el primer grupo, debido fundamentalmente a los bajos niveles de ansiedad que los deberes escolares le generan.

Pero los niveles más altos de ansiedad ante los deberes escolares son los del grupo de evitación del fracaso. El temor al fracaso, que caracteriza a los estudiantes con este perfil motivacional, los lleva a una constante preocupación por evitar parecer incompetentes ante los demás. A pesar de que esto puede generar beneficios para la propia imagen, suele tener consecuencias muy negativas para la implicación y el compromiso del estudiante en los deberes escolares, ya que lo más probable es que se impliquen lo menos posible o, incluso, dejen de implicarse por el miedo a fracasar y por la ansiedad que les provocan los deberes. Estos resultados coinciden, en general, con las aportaciones de otros estudios en los que se encontró una relación positiva entre metas de evitación y ansiedad (e.g., Middleton y Midgley, 1997)

El grupo de estudiantes desmotivados es el que presenta los niveles de ansiedad más bajos. Sus niveles motivacionales son tan bajos que no sólo les lleva a una falta de implicación en los deberes escolares y un bajo rendimiento académico sino también a que los niveles de ansiedad provocados por los deberes sean también los más bajos. En definitiva, parece esperable que a este grupo de estudiantes al no tener motivos de peso para implicarse, no les generen un alto grado de ansiedad ni los deberes ni sus bajos resultados académicos.

Por lo tanto, los resultados indican que el grupo "más deseable" sería el que posee el perfil de metas orientadas al aprendizaje. Precisamente, son estas metas las que están relacionadas positivamente con numerosos resultados educativos favorables, tales como el uso de estrategias que contribuyen a un aprendizaje comprensivo, una alta persistencia, interés y motivación intrínseca (Hulleman. Schrager, Bodmann y Harackiewicz, 2010). En consecuencia, una orientación motivacional hacia metas de aprendizaje, ayudará al estudiante a mantener su sentido de eficacia personal frente a las experiencias de fracaso, le protegerá ante factores negativos como la ansiedad y le facilitará un mayor nivel de compromiso cognitivo $y$, en consecuencia, un mejor rendimiento (Valle et al., en prensa).

A esto habría que añadir que, tal y como se demuestra en el presente trabajo, el grupo de metas de aprendizaje no sólo se implica más y mejor en los deberes escolares sino que también obtiene mejores resultados académicos que el resto de los grupos. De esta forma, si un estudiante está motivado para aprender, realizará mayor cantidad de deberes, les dedicará más tiempo y gestionará mejor ese tiempo $\mathrm{y}$, como consecuencia, también logrará un mejor rendimiento. Además, es muy probable que adopten un enfoque de trabajo más profundo, implicándose en los deberes con la intención de aprender y reforzar los conocimientos adquiridos en clase, preocupándose por solventar las dudas que se les plantean durante su realización y relacionando los deberes con lo aprendido previamente.

\section{Referencias}

Cohen, J. (1988). Statistical power analysis for the behavioral sciences ( $2^{\mathrm{a}}$ ed.). Hillsdale, NJ: Erlbaum

Dweck, C. S. (2001). Motivación social: metas y procesos sociocognitivos. Un comentario. En J. Juvonen y K. R. Wentzel (Eds), Motivación y adaptación escolar (pp. 217-234). México: Oxford University Press.

Hair, J. E., Anderson, R. E., Tatham, R. L. y Black W. C. (1999). Multivariate data analysis. (6 $6^{\mathrm{a}}$ ed.). Upper Saddle River, NJ: Prentice-Hall.

Harackiewicz, J. M. y Linnenbrink, E. A. (2005). Multiple Achievement Goals and Multiple Pathways for Learning: The Agenda and Impact of Paul R. Pintrich. Educational Psychologist, 40(2), 75-84.

Hulleman, C. S., Schrager, S. M., Bodmann, S. M. y Harackiewicz, J. M. (2010). A meta-analytic review of achievement goal measures: Different labels for the same constructs or different constructs with similar labels? Psychological Bulletin, 136, 422-449.

Mestre, V., Samper, P., Tur, A.M., Cortés, M. T. y Nácher, M. J. (2006). Conducta prosocial y procesos psicológicos implicados: Un estudio longitudinal en la 
adolescencia. Revista Mexicana de Psicología, 23, 203-216

Middleton, M. y Midgley, C. (1997). Avoiding the demonstration of lack of ability: An under-explored aspect of goal theory. Journal of Educational Psychology, 89, 710-718.

Núñez, J. C., González-Pienda, J. A., GonzálezPumariega, S, García, M., y Roces, C. (1997). Cuestionario para la Evaluación de Metas Académicas en Secundaria (CEMA-II). Departamento de Psicología. Universidad de Oviedo.

Núñez, J. C., Suárez, N., Cerezo, R., González-Pienda, J. A., Rosário, P., Mourao, R. y Valle, A. (2013). Homework and academic achievement across Spanish Compulsory Education. Educational Psychology. doi: 10.1080/01443410.2013.817537

Núñez, J. C., Suárez, N., Rosário, P., Vallejo, G., Cerezo, R., y Valle, A. (2015). Teachers' feedback on homework, homework-related behaviors and academic achievement. The Journal of Educational Research, 108(3), 204-216.

Pan, I., Regueiro, B., Ponte, B., Rodríguez, S., Piñeiro, I., y Valle, A. (2013). Motivación, implicación en los deberes escolares y rendimiento académico. Aula Abierta, 41(3), 13-22.

Rosário, P., Mourão, R., Baldaque, M., Nunes, T., Núñez, J. C., González-Pienda, J.A., Cerezo, R. y Valle, A. (2009). Tareas para casa, autorregulación del aprendizaje y rendimiento en matemáticas. Revista de Psicodidáctica, 14, 179-192.

Suárez, J. M., Cabanach, R. G. y Valle, A. (2001). Multiple-goal pursuit and its relation to cognitive, self-regulatory, and motivational strategies. British Journal of Educational Psychology, 71, 561-572.
Sun, Sh., Pan, W. y Wang, L. L. (2010). A comprehensive review of effect size reporting and interpreting practices in academic journals in education and psychology. Journal of Educational Psychology, 102, 989-1004.

Valle, A., Cabanach, R. G., Núñez, J. C., GonzálezPienda, J., Rodríguez, S. y Piñeiro, I. (2003). Multiple goals, motivation and academic learning. British Journal of Educational Psychology, 73, 71-87.

Valle, A., Núñez, J. C., Cabanach, R .G., Rodríguez, S., Rosário, P. e Inglés, C. (2013). Motivational profiles as a combination of academic goals in higher education. Educational Psychology. doi: 10.1080/01443410.2013.819072

Valle, A., Pan, I., Núñez, J. C., Rosário, P., Rodríguez, S. y Regueiro, B. (2015). Deberes escolares y rendimiento académico en Educación Primaria. Anales de Psicología, 31(2), 562-569.

Valle, A., Regueiro, B., Rodríguez, S., Piñeiro, I., Freire, C., Ferradás, M. y Suárez, N. (en prensa). Perfiles motivacionales como combinación de expectativas de autoeficacia y metas académicas en estudiantes universitarios. European Journal of Education and Psychology.

Wentzel, K. R. (2001). Metas sociales y relaciones sociales como motivadores de la adaptación a la escuela: un análisis motivacional. En J. Juvonen y K. R. Wentzel (Eds.), Motivación y adaptación escolar (pp. 269-294). México: Oxford University Press.

Zimmerman, B. J., Bonner, S. y Kovach, R. (1996). Developing selfregulated learners: Beyond achievement to self-efficacy. Washington, DC: American Psychological Association. 